\title{
ILL-POSED PROBLEM OF RECONSTRUCTION OF THE POPULATION SIZE IN THE HUTCHINSON-WRIGHT EQUATION $^{1,2}$
}

\author{
Yurii F. Dolgii \\ Ural Federal University and N.N. Krasovskii Institute of Mathematics and Mechanics of \\ the Ural Branch of the Russian Academy of Sciences, Ekaterinburg, Russia, \\ Yurii.Dolgii@usu.ru \\ Platon G. Surkov \\ Ural Federal University and N.N. Krasovskii Institute of Mathematics and Mechanics of \\ the Ural Branch of the Russian Academy of Sciences, Ekaterinburg, Russia, \\ platon.surkov@gmail.com
}

\begin{abstract}
We consider an ill-posed problem of reconstruction of the population size in the Hutchinson-Wright Equation. Regularized solutions were constructed on the finite interval of the negative half-line.

Keywords: The Hutchinson-Wright equation, Ill-posed problem, Asymptotic methods.
\end{abstract}

\section{Statement of the Problem}

Consider the population model represented by the Hutchinson-Wright equation

$$
\frac{d N(t)}{d t}=r\left(1-\frac{1}{k} N(t-h)\right) N(t), \quad t \in \mathbb{R}
$$

where $N: \mathbb{R} \rightarrow \mathbb{R}^{+}=(0,+\infty)$ is the population size, $r$ is the intrinsic growth rate (the birth rate), $k$ is the carrying capacity, $h$ is the delay parameter (the breeding age).

The work [1] is devoted to the investigation of various biological factors affecting changes in the number of population. In the analysis of regularities of the change of population the results of observations of the size of biological populations [2] are used. Full-scale observation of changes of population size requires significant material and labor costs. Mathematical modeling can facilitate solution of the problem of studying the dynamics of the quantitative change of population size [3].

The Hutchinson-Wright equation describes a mathematical model of the single-species biocenosis, when the influence of predators is slight, the habitat is homogenous, migration processes do not have a significant impact on the change of population size and the quantity of available food is restored regularly up to a certain level.

The works $[3,4]$ are devoted to the investigation of various qualitative problems of the change of population size for the Hutchinson's equation. The computer simulation of such changes was held in [5]. The motivation of these works is joint with the forecast of changes of population size. The correctness of the initial problem for the Hutchinson's equation helps to solve this problem successfully. The main qualitative result is that the population size tends to constant when time increase or periodic oscillations take place. It is depend on the value of parameter $h$. If the population is on the stage of a transient of changes of the population size, the inverse problem of

\footnotetext{
${ }^{1}$ This work was supported in part by the Program on Basic Scientific Research of the UrB of the Russian Acad. Sci. (no. 15-16-1-8) and by the Russian Foundation for Basic Research (project no. 13-01-00094).

${ }^{2}$ Published in Russian in Trudy Inst. Mat. i Mekh. UrO RAN, 2011. Vol. 17. No 1. P. 70-84.
} 
determining its size in the previous periods of time is of interest. From the mathematical point of view this problem is more difficult, because it belongs to a class of ill-posed problems [6].

In the present paper the ill-posed problem of reconstruction of the population size in the Hutchinson-Wright equation is solved.

It is supposed that an information about the population size is known on the time interval $\left[t_{0}-h, t_{0}\right]$. In the sequel, without loss the generality we assume that $t_{0}=0$. The population size on the interval $[-h, 0]$ is defined by a positive function $\varphi$ which belongs to a separable Hilbert space $H=L_{2}[-h, 0) \times \mathbb{R}$ with inner product $(\varphi, \psi)=\psi(0) \varphi(0)+\int_{-h}^{0} \psi(s) \varphi(s) d s$. When reconstructing the number of population we use the method of steps in the direction of decreasing time. Then for the finding functions $x_{m}(\vartheta)=N(m h+\vartheta), \vartheta \in[-h, 0], m \leq-1$, we have a system of equations

$$
U\left(x_{m}\right)=x_{m+1}, \quad m \leq-1, \quad x_{0}=\varphi,
$$

where the operator $U: H \rightarrow H$ is determined by the formula

$$
U(x)(\vartheta)=\exp \left(r(h+\vartheta)-\frac{r}{k} \int_{-h}^{\vartheta} x(s) d s\right) x(0), \quad \vartheta \in[-h, 0] .
$$

Thus, the reconstruction of population size is associated with solving the ill-posed problem

$$
U(x)=\varphi .
$$

\section{Determining System of Equations for Finding the Values of the Regularizing Operator}

We use the regularization method of A.N. Tikhonov to solve the ill-posed problem. So we choose a stabilizing functional of the form

$$
\Omega[x]=G x^{2}(0)+\int_{-h}^{0}\left(Q x^{2}(s)+P x^{2}(s)\right) d s, \quad x \in W_{2}^{1}[-h, 0],
$$

where $G, P$, and $Q$ are positive numbers, $x^{\prime}$ is the derivative of the function $x$. One needs to find an element $x_{\alpha} \in W_{2}^{1}[-h, 0]$ minimizing the smoothing functional

$$
M^{\alpha}[\varphi, x]=(U(x)-\varphi, U(x)-\varphi)+\alpha \Omega[x], \quad x \in W_{2}^{1}[-h, 0],
$$

for a fixed positive value of the regularization parameter $\alpha$.

We obtain the necessary condition for a minimum of the functional [7, p. 113] when finding the Gâteaux derivative of the smoothing functional

$$
\begin{aligned}
M_{x}^{\alpha}[\varphi, y]^{\prime} & =\lim _{\mu \rightarrow 0} \mu^{-1}\left(\left(U(x)-\varphi+\mu U_{x}^{\prime}(x) y, U(x)-\varphi+\mu U_{x}^{\prime}(x) y\right)+\alpha \Omega[x+\mu y]\right. \\
& \left.-(U(x)-\varphi, U(x)-\varphi)-\alpha \Omega[x]+O\left(\mu^{2}\right)\right)=\left(U(x)-\varphi, U_{x}^{\prime}(x) y\right) \\
& +\alpha\left(y(0) G x(0)+\int_{-h}^{0}\left(y(s) Q x(s)+y^{\prime}(s) P x^{\prime}(s)\right) d s\right) .
\end{aligned}
$$

As a result we have

$$
\left(U_{x}^{\prime *}(x) U(x)-U_{x}^{\prime *}(x) \varphi, y\right)+\alpha\left(y(0) G x(0)+\int_{-h}^{0}\left(y(s) Q x(s)+y^{\prime}(s) P x^{\prime}(s)\right) d s\right)=0 .
$$


Here the Gâteaux derivative of the operator $U$ in the point $x$ determined by the formula

$$
\left(U_{x}^{\prime}(x) y\right)(\vartheta)=\exp \left(r(h+\vartheta)-\frac{r}{k} \int_{-h}^{\vartheta} x(s) d s\right)\left(y(0)-\frac{r}{k} x(0) \int_{-h}^{\vartheta} y(s) d s\right), \quad \vartheta \in[-h, 0] .
$$

The adjoint operator $U_{x}^{\prime *}(x)$ given by the formulas

$$
\left(U_{x}^{\prime *}(x) y\right)(\vartheta)= \begin{cases}\exp \left(r h-\frac{r}{k} \int_{-h}^{0} x(s) d s\right)(y(0) & \\ \left.+\int_{-h}^{0} \exp \left(r s-\frac{r}{k} \int_{0}^{s} x\left(s_{1}\right) d s_{1}\right) y(s) d s\right), & \vartheta=0, \\ -\frac{r}{k} x(0) \exp \left(r h-\frac{r}{k} \int_{-h}^{0} x(s) d s\right)(y(0) & \\ \left.+\int_{\vartheta}^{0} \exp \left(r s-\frac{r}{k} \int_{0}^{s} x\left(s_{1}\right) d s_{1}\right) y(s) d s\right), & \vartheta \in[-h, 0) .\end{cases}
$$

By using the definition of inner product of the space $H$, we reduce the necessary condition for the minimum of the smoothing functional to the form

$$
\begin{gathered}
y(0)\left(\left(U_{x}^{\prime *}(x) U(x)\right)(0)-\left(U_{x}^{\prime *}(x) \varphi\right)(0)\right)+\int_{-h}^{0} y(s)\left(\left(U_{x}^{\prime *}(x) U(x)\right)(s)-\left(U_{x}^{\prime *}(x) \varphi\right)(s)\right) d s \\
+\alpha y(0) G x(0)+\alpha \int_{-h}^{0}\left(y(s) Q x(s)+y^{\prime}(s) P x^{\prime}(s)\right) d s=0, \quad x \in W_{2}^{1}[-h, 0],
\end{gathered}
$$

which must be valid for any $y \in W_{2}^{1}[-h, 0]$. For the functions $x \in W_{2}^{2}[-h, 0]$ we reduce the integral of the last summand by applying the integration by parts formula. Then the condition (2.1) takes the form

$$
\begin{gathered}
y(0)\left(\left(U_{x}^{\prime *}(x) U(x)\right)(0)-\left(U_{x}^{\prime *}(x) \varphi\right)(0)+\alpha\left(G x(0)+P x^{\prime}(0)\right)\right)-\alpha y(-h) P x^{\prime}(-h) \\
+\int_{-h}^{0} y(s)\left(\left(U_{x}^{\prime *}(x) U(x)\right)(s)-\left(U_{x}^{\prime *}(x) \varphi\right)(s)+\alpha\left(Q x(s)-P x^{\prime \prime}(s)\right)\right) d s=0
\end{gathered}
$$

and must be valid for any $y \in H$. A possible minimizing element $x_{\alpha}$ satisfies the system of equations

$$
\begin{array}{rlrl}
\left(U_{x}^{\prime *}(x) U(x)\right)(\vartheta)+\alpha\left(Q x(\vartheta)-P x^{\prime \prime}(\vartheta)\right) & =\left(U_{x}^{\prime *}(x) \varphi\right)(\vartheta), & \vartheta \in[-h, 0), \\
\left(U_{x}^{\prime *}(x) U(x)\right)(0)+\alpha\left(G x(0)+P x^{\prime}(0)\right) & =\left(U_{x}^{\prime *}(x) \varphi\right)(0), & & x^{\prime}(-h)=0 .
\end{array}
$$

By introducing auxiliary functions $\psi$ and $\chi$ by the formulas

$$
\begin{array}{lll}
\psi(\vartheta)=\left(U_{x}^{*}(x)(\chi-\varphi)\right)(\vartheta), & \vartheta \in[-h, 0), & \psi(0)=\psi(-0), \\
\chi(\vartheta)=(U x)(\vartheta), & \vartheta \in[-h, 0], &
\end{array}
$$

and by using the representations of the operators $U$ and $U_{x}^{\prime *}(x)$, one can replace system of equations for the minimizing element by an equivalent boundary value problem for ordinary differential equations. 
Assertion 1. The possible minimizing element $x_{\alpha}$ is a component of the solution of the following system of ordinary differential equations

$$
\begin{aligned}
x^{\prime \prime} & =P^{-1} Q x+\alpha^{-1} P^{-1} \psi, \\
\psi^{\prime} & =\frac{r}{k} \chi(\chi-\varphi(\vartheta)), \\
\chi^{\prime} & =r\left(1-\frac{1}{k} x\right) \chi
\end{aligned}
$$

with the boundary conditions

$$
\begin{gathered}
x^{\prime}(-h)=0, \quad \psi(-h)-\alpha \frac{r}{k} x(0)\left(G x(0)+P x^{\prime}(0)\right)=0, \\
\psi^{\prime}(0)+\psi(0)=0, \quad x(0)=\chi(-h) .
\end{gathered}
$$

Here $\varphi \in H$, the component $\chi_{\alpha}$ of the solution of the boundary value problem (2.3), (2.4) satisfies the condition $\chi_{\alpha}=U x_{\alpha}$, and $\alpha$ is a small positive parameter.

$\mathrm{P} \mathrm{r}$ o o f. By using the auxiliary function $\psi$ and continuing the first equation of the system (2.2) to $[-h, 0]$, we obtain

$$
\psi(\vartheta)+\alpha\left(Q x(\vartheta)-P x^{\prime \prime}(\vartheta)\right)=0, \quad \vartheta \in[-h, 0] .
$$

As it appears from the definition of the function $\chi$ it satisfies to the differential equation

$$
\chi^{\prime}(\vartheta)=r \chi(\vartheta)\left(1-\frac{1}{k} x(\vartheta)\right), \quad \vartheta \in[-h, 0],
$$

with the boundary condition $\chi(-h)=x(0)$.

Once again, from the definition of the function $\psi$ we have that it satisfies to the differential equation

$$
\psi^{\prime}(\vartheta)=\frac{r}{k} \chi(\vartheta)(\chi(\vartheta)-\varphi(\vartheta)), \quad \vartheta \in[-h, 0],
$$

with the boundary condition

$$
\psi(0)=\psi(-0)=-\frac{r}{k} \chi(0)(\chi(0)-\varphi(0))=-\psi^{\prime}(0) .
$$

By using the auxiliary functions we rewrite the second equation of the system (2.2)

$$
\begin{gathered}
\exp \left(r h-\frac{r}{k} \int_{-h}^{0} x(s) d s\right)(\chi(0) \\
\left.-\varphi(0)+\int_{-h}^{0} \exp \left(r s-\frac{r}{k} \int_{0}^{s} x\left(s_{1}\right) d s_{1}\right)(\chi(s)-\varphi(s)) d s\right) \\
+\alpha\left(G x(0)+P x^{\prime}(0)\right)=0 .
\end{gathered}
$$

By taking into account the value of the auxiliary function $\psi(-h)$, we reduce the last equation to the form

$$
\psi(-h)-\alpha \frac{r}{k} x(0)\left(G x(0)+P x^{\prime}(0)\right)=0 .
$$

Let us eliminate the auxiliary variables $\psi$ and $\chi$ from the system of equations (2.3) and the bounadry conditions (2.4). When finding a solution of this problem we impose additional conditions on the initial function and solution of the boundary value problem, such as $\varphi \in W_{2}^{1}[-h, 0]$ and $\max _{\vartheta \in[-h, 0]}|\chi(\vartheta)-\varphi(\vartheta)|$ is a small value. The last restriction is compatible with a statement of the problem of regularization and allows to find the following

$$
\chi=\frac{1}{2}\left(\varphi(\vartheta)+\sqrt{\varphi^{2}(\vartheta)+4 \frac{k}{r} \psi^{\prime}}\right),
$$


from the second equation of the system (2.3), as well as guarantee the fulfillment of the condition $\min _{\vartheta \in[-h, 0]}\left(\varphi^{2}(\vartheta)+(4 k / r) \psi^{\prime}(\vartheta)\right)>0$.

By calculating a derivative of the last expression and by substituting it into the third equation of the system (2.3), we obtain

$$
\begin{gathered}
\varphi^{\prime}(\vartheta)+\left(\varphi^{2}(\vartheta)+4 \frac{k}{r} \psi^{\prime}\right)^{-1 / 2}\left(\varphi(\vartheta) \varphi^{\prime}(\vartheta)+2 \frac{k}{r} \psi^{\prime \prime}\right) \\
=\left(\varphi(\vartheta)+\left(\varphi^{2}(\vartheta)+4 \frac{k}{r} \psi^{\prime}\right)^{1 / 2}\right)\left(r-\frac{r}{k} x\right) .
\end{gathered}
$$

When differentiating the first equation of the system (2.3) twice, we find

$$
\psi^{\prime}=\alpha P x^{\prime \prime \prime}-\alpha Q x^{\prime}, \quad \psi^{\prime \prime}=\alpha P x^{\mathrm{IV}}-\alpha Q x^{\prime \prime} .
$$

By eliminating $\psi$ from equation (2.6) and by introducing variables $x_{1}=x, x_{2}=\alpha^{1 / 4} x^{\prime}, x_{3}=\alpha^{1 / 2} x^{\prime \prime}$, $x_{4}=\alpha^{3 / 4} x^{\prime \prime \prime}$, we obtain the system of differential equations

$$
\begin{gathered}
\alpha^{1 / 4} x_{1}^{\prime}=x_{2}, \quad \alpha^{1 / 4} x_{2}^{\prime}=x_{3}, \quad \alpha^{1 / 4} x_{3}^{\prime}=x_{4}, \\
\alpha^{1 / 4} x_{4}^{\prime}=\frac{r}{2 k P}\left(\varphi^{2}(\vartheta)+4 \frac{k}{r}\left(\alpha^{1 / 4} P x_{4}-\alpha^{3 / 4} Q x_{2}\right)\right)^{1 / 2}\left(\varphi(\vartheta)\left(r-\frac{r}{k} x_{1}\right)-\varphi^{\prime}(\vartheta)\right) \\
+\alpha^{1 / 2} \frac{Q}{P} x_{3}+\frac{r}{2 k P}\left(\varphi^{2}(\vartheta)+4 \frac{k}{r}\left(\alpha^{1 / 4} P x_{4}-\alpha^{3 / 4} Q x_{2}\right)\right)\left(r-\frac{r}{k} x_{1}\right)-\frac{r}{2 k P} \varphi(\vartheta) \varphi^{\prime}(\vartheta) .
\end{gathered}
$$

We pass the above-introduced new variables in the boundary conditions (2.4). So we have

$$
\begin{gathered}
x_{2}(-h)=0, \quad P x_{4}(0)-\alpha^{1 / 2} Q x_{2}(0)+\alpha^{1 / 4} P x_{3}(0)-\alpha^{3 / 4} Q x_{1}(0)=0, \\
P x_{3}(-h)-\alpha^{1 / 2} Q x_{1}(-h)-\alpha^{1 / 4} \frac{r}{k} P x_{1}(0) x_{2}(0)-\alpha^{1 / 2} \frac{r}{k} G x_{1}^{2}(0)=0, \\
2 x_{1}(0)-\varphi(-h)-\left(\varphi^{2}(-h)+4 \frac{r}{k}\left(\alpha^{1 / 4} P x_{4}(-h)-\alpha^{3 / 4} Q x_{2}(-h)\right)\right)^{1 / 2}=0 .
\end{gathered}
$$

By introducing the vector $X=\left\|x_{j}\right\|_{1}^{4}$, we rewrite the system (2.7) in the vector form

$$
\frac{d X}{d \vartheta}=\alpha^{-1 / 4} \mathcal{A}(\vartheta) X+\alpha^{-1 / 4} \widetilde{\Phi}_{1}(\vartheta)+\widetilde{\Phi}_{2}(\vartheta, \alpha, X),
$$

where

$$
\begin{gathered}
\mathcal{A}(\vartheta)=\left(\begin{array}{cccc}
0 & 1 & 0 & 0 \\
0 & 0 & 1 & 0 \\
0 & 0 & 0 & 1 \\
-r^{2} \varphi^{2}(\vartheta) /\left(k^{2} P\right) & 0 & 0 & 0
\end{array}\right), \quad \widetilde{\Phi}_{1}^{\top}(\vartheta)=\left(0,0,0, r \varphi(\vartheta)\left(r \varphi(\vartheta)-\varphi^{\prime}(\vartheta)\right) /(k P)\right), \\
\widetilde{\Phi}_{2}^{\top}(\vartheta, \alpha, X)=\left(0,0,0, \frac{2\left(r \varphi(\vartheta)\left(1-(1 / k) x_{1}\right)-\varphi^{\prime}(\vartheta)\right)\left(P x_{4}-\alpha^{1 / 2} Q x_{2}\right)}{\left(\varphi^{2}(\vartheta)+(4 k / r)\left(\alpha^{1 / 4} P x_{4}-\alpha^{3 / 4} Q x_{2}\right)\right)^{1 / 2}+\varphi(\vartheta)}+\alpha^{1 / 4} \frac{Q}{P} x_{3}\right. \\
\left.+\frac{2 r}{P}\left(P x_{4}-\alpha^{1 / 2} Q x_{2}\right)\left(1-\frac{1}{k} x_{1}\right)\right) .
\end{gathered}
$$

Let us make a replacement of variables in the system (2.9):

$$
X=T(\vartheta) y, \quad \vartheta \in[-h, 0],
$$


where $T(\vartheta)=\left\|t_{i j}(\vartheta)\right\|_{1}^{4}=\left\|e_{j}^{i-1} \lambda^{i-1}(\vartheta)\right\|_{1}^{4}$ is a matrix reducing $\mathcal{A}(\vartheta)$ to the Jordan form, $\lambda(\vartheta)=$ $(r \varphi(\vartheta) /(k \sqrt{P}))^{1 / 2}, e_{1}=\bar{e}, e_{2}=e, e_{3}=-\bar{e}, e_{4}=-e$, and $e=(1+i) / \sqrt{2}$.

Then we obtain the system

$$
\frac{d y}{d \vartheta}=\alpha^{-1 / 4} J(\vartheta) y+\alpha^{-1 / 4} \Phi_{1}(\vartheta)+\Phi_{2}(\vartheta, \alpha, y),
$$

where

$$
\begin{gathered}
J(\vartheta)=\operatorname{diag}\left(e_{1} \lambda(\vartheta), e_{2} \lambda(\vartheta), e_{3} \lambda(\vartheta), e_{4} \lambda(\vartheta)\right), \\
\Phi_{1}(\vartheta)=T^{-1}(\vartheta) \widetilde{\Phi}_{1}(\vartheta)=r /(4 k P) \lambda^{-3}(\vartheta) \varphi(\vartheta)\left(r \varphi(\vartheta)-\varphi^{\prime}(\vartheta)\right)\left\|e_{j}^{-3}\right\|_{1}^{4}, \\
\Phi_{2}(\vartheta, \alpha, y)=T^{-1}(\vartheta)\left(\widetilde{\Phi}_{2}(\vartheta, \alpha, T(\vartheta) y)-T^{\prime}(\vartheta) y\right), \quad \vartheta \in[-h, 0] .
\end{gathered}
$$

Theorem 1. Let $\varphi \in W_{\infty}^{2}[-h, 0]$. Then the components of the solution of the system (2.11) are determined by the asymptotic formulas

$$
\begin{gathered}
y_{j}(\vartheta, \alpha, D)=\exp \left(\alpha^{-1 / 4} e_{j} \int_{\vartheta_{j}}^{\vartheta} \lambda(\tau) d \tau\right) D_{j}-e_{j}^{-1} \lambda^{-1}(\vartheta) \Phi_{1}^{j}(\vartheta) \\
+O\left(\alpha^{1 / 4} ; \vartheta, D_{1}, \ldots, D_{4}\right), \quad j=\overline{1,4}, \quad \vartheta \in[-h, 0]
\end{gathered}
$$

where $D=\left\|D_{s}\right\|_{1}^{4}, D_{s}, s=\overline{1,4}$ are arbitrary vectors belonging to $\mathbb{C}^{n}$.

P r o o f. Solutions of the system

$$
\frac{d y}{d \vartheta}=\alpha^{-1 / 4} J(\vartheta) y
$$

are defined by the formulas

$$
y_{j}\left(\vartheta, \alpha, \widehat{D}_{j}\right)=\exp \left(\alpha^{-1 / 4} e_{j} \int_{\vartheta_{j}}^{\vartheta} \lambda(\tau) d \tau\right) \widehat{D}_{j}, \quad \vartheta \in[-h, 0], \quad j=\overline{1,4},
$$

where $\widehat{D}_{j}$ are arbitrary constants belonging to $\mathbb{C}^{n}, \vartheta_{1}=\vartheta_{2}=0$, and $\vartheta_{3}=\vartheta_{4}=-h$.

By using the method of variation of constants for the nonlinear system (2.11), we have

$$
\widehat{D}_{j}^{\prime}(\vartheta)=\exp \left(-\alpha^{-1 / 4} e_{j} \int_{\vartheta_{j}}^{\vartheta} \lambda(\tau) d \tau\right)\left(\alpha^{-1 / 4} \Phi_{1}^{j}(\vartheta)+\Phi_{2}^{j}(\vartheta, \alpha, y(\vartheta, \alpha, \widehat{D}(\vartheta)))\right), \quad j=\overline{1,4} .
$$

By integrating the last equalities, we obtain

$$
\begin{gathered}
\widehat{D}_{j}(\vartheta)=\widetilde{D}_{j}+\int_{\vartheta_{j}}^{\vartheta} \exp \left(-\alpha^{-1 / 4} e_{j} \int_{\vartheta_{j}}^{s} \lambda(\tau) d \tau\right)\left(\alpha^{-1 / 4} \Phi_{1}^{j}(s)+\Phi_{2}^{j}(s, \alpha, y(s, \alpha, \widehat{D}(s)))\right) d s, \\
\vartheta \in[-h, 0], \quad j=\overline{1,4},
\end{gathered}
$$

where $\widetilde{D}_{j}$ are new constants.

By substituting the last expression into (2.13), we find

$$
y_{j}\left(\vartheta, \alpha, \widehat{D}_{j}(\vartheta)\right)=\exp \left(\alpha^{-1 / 4} e_{j} \int_{\vartheta_{j}}^{\vartheta} \lambda(\tau) d \tau\right) \widetilde{D}_{j}+\mathcal{I}_{1}+\mathcal{I}_{2}, \quad \vartheta \in[-h, 0], \quad j=\overline{1,4},
$$


where

$$
\begin{gathered}
\mathcal{I}_{1}=\alpha^{-1 / 4} \int_{\vartheta_{j}}^{\vartheta} \exp \left(\alpha^{-1 / 4} e_{j} \int_{s}^{\vartheta} \lambda(\tau) d \tau\right) \Phi_{1}^{j}(s) d s \\
\left.\mathcal{I}_{2}=\int_{\vartheta_{j}}^{\vartheta} \exp \left(\alpha^{-1 / 4} e_{j} \int_{s}^{\vartheta} \lambda(\tau) d \tau\right) \Phi_{2}^{j}(s, \alpha, y(s, \alpha, \widehat{D}(s)))\right) d s .
\end{gathered}
$$

By applying the integration by parts formula to $\mathcal{I}_{1}$, we have

$$
\mathcal{I}_{1}=-\left(e_{j} \lambda(\vartheta)\right)^{-1} \Phi_{1}^{j}(\vartheta)+\exp \left(\alpha^{-1 / 4} e_{j} \int_{\vartheta_{j}}^{\vartheta} \lambda(\tau) d \tau\right)\left(e_{j} \lambda\left(\vartheta_{j}\right)\right)^{-1} \Phi_{1}^{j}\left(\vartheta_{j}\right)+O\left(\alpha^{1 / 4} ; \vartheta\right)
$$

By substituting the last formula into (2.14), we obtain a system of nonlinear integral equations

$$
\begin{gathered}
\hat{y}_{j}(\vartheta)=f_{j}\left(\vartheta, \alpha, D_{j}\right)+\int_{\vartheta_{j}}^{\vartheta} \exp \left(\alpha^{-1 / 4} e_{j} \int_{s}^{\vartheta} \lambda(\tau) d \tau\right) \Phi_{2}^{j}(s, \alpha, \hat{y}(s)) d s=(A \hat{y})_{j}(\vartheta), \\
\vartheta \in[-h, 0], \quad j=\overline{1,4},
\end{gathered}
$$

where

$$
\begin{gathered}
\hat{y}_{j}(\vartheta)=y_{j}\left(\vartheta, \alpha, \widehat{D}_{j}(\vartheta)\right) \\
f_{j}\left(\vartheta, \alpha, D_{j}\right)=\exp \left(\alpha^{-1 / 4} e_{j} \int_{\vartheta_{j}}^{\vartheta} \lambda(\tau) d \tau\right) D_{j}-\left(e_{j} \lambda(\vartheta)\right)^{-1} \Phi_{1}^{j}(\vartheta)+O\left(\alpha^{1 / 4} ; \vartheta\right) \\
D_{j}=\widetilde{D}_{j}+\left(e_{j} \lambda\left(\vartheta_{j}\right)\right)^{-1} \Phi_{1}^{j}\left(\vartheta_{j}\right)
\end{gathered}
$$

are new arbitrary constants.

By using the principle of contraction mapping [8], we will show that the system of equations $(2.15)$ has a unique solution in the space $L_{\infty}\left([-h, 0], \mathbb{R}^{n}\right)$ for small values of $\alpha$ on the set $\Omega=\left\{\hat{y}(\cdot):\|\hat{y}(\cdot)-f(\cdot, \alpha, D)\|_{\infty} \leq \varepsilon\right\}$. Here $f(\cdot, \alpha, D)=\left\|f_{j}\left(\cdot, \alpha, D_{j}\right)\right\|_{1}^{4}, D=\left\|D_{j}\right\|_{1}^{4}$, $\|\hat{y}(\cdot)\|_{\infty}=$ vrai $\sup _{\vartheta \in[-h, 0]} \max _{1 \leq j \leq 4}\left|\hat{y}_{j}(\vartheta)\right|$, and $\varepsilon$ is a some small positive number. Indeed, for small values of $\alpha$ the condition $A \Omega \subseteq \Omega$ is fulfilled because

$$
\begin{gathered}
\left|(A \hat{y})_{j}(\vartheta)-f_{j}\left(\vartheta, \alpha, D_{j}\right)\right| \leq \operatorname{vrai} \sup _{\vartheta \in[-h, 0]} \max _{1 \leq j \leq 4}\left|\int_{\vartheta_{j}}^{\vartheta} \lambda(s) \exp \left(\alpha^{-1 / 4} \operatorname{Re} e_{j} \int_{s}^{\vartheta} \lambda(\tau) d \tau\right) d s\right| \Psi \\
\leq \Psi \sqrt{2} \alpha^{1 / 4} \text { vrai } \sup _{\vartheta \in[-h, 0]} \max _{1 \leq j \leq 4}\left(1-\exp \left(\alpha^{-1 / 4} \operatorname{Re} e_{j} \int_{\vartheta_{j}}^{\vartheta} \lambda(\tau) d \tau\right)\right) \leq \sqrt{2} \alpha^{1 / 4} \Psi,
\end{gathered}
$$

where $\Psi=$ vrai $\sup _{s \in[-h, 0]} \max _{1 \leq j \leq 4}\left|\Phi_{2}^{j}(s, \alpha, \hat{y}(s)) / \lambda(s)\right|$.

By using the Lipschitz condition

$$
\left|\Phi_{2}^{j}\left(s, \alpha, \hat{y}_{1}(s)\right)-\Phi_{2}^{j}\left(s, \alpha, \hat{y}_{2}(s)\right)\right| \leq L\left|\hat{y}_{1}(s)-\hat{y}_{2}(s)\right|, \quad s \in[-h, 0], \quad 1 \leq j \leq 4,
$$

we obtain

$$
\begin{gathered}
\left|\left(A \hat{y}_{1}\right)_{j}(\vartheta)-\left(A \hat{y}_{2}\right)_{j}(\vartheta)\right| \\
\leq \operatorname{vrai} \sup _{\vartheta \in[-h, 0]} \max _{1 \leq j \leq 4}\left|\int_{\vartheta_{j}}^{\vartheta} \exp \left(\alpha^{-1 / 4} \operatorname{Re} e_{j} \int_{s}^{\vartheta} \lambda(\tau) d \tau\right)\left(\Phi_{2}^{j}\left(s, \alpha, \hat{y}_{1}(s)\right)-\Phi_{2}^{j}\left(s, \alpha, \hat{y}_{2}(s)\right)\right) d s\right|
\end{gathered}
$$




$$
\begin{gathered}
\leq L \text { vrai } \sup _{\vartheta \in[-h, 0]} \max _{1 \leq j \leq 4}\left|\hat{y}_{1}(s)-\hat{y}_{2}(s)\right| \text { vrai } \sup _{\vartheta \in[-h, 0]} \max _{1 \leq j \leq 4}\left|\int_{\vartheta_{j}}^{\vartheta} \exp \left(\alpha^{-1 / 4} \operatorname{Re} e_{j} \int_{s}^{\vartheta} \lambda(\tau) d \tau\right) d s\right| \\
\leq \frac{\sqrt{2} L \alpha^{1 / 4}}{\min _{\vartheta \in[-h, 0]} \lambda(\vartheta)} \text { vrai } \sup _{\vartheta \in[-h, 0]} \max _{1 \leq j \leq 4}\left(1-\exp \left(\alpha^{-1 / 4} \operatorname{Re} e_{j} \int_{\vartheta_{j}}^{\vartheta} \lambda(\tau) d \tau\right)\right)\left\|\hat{y}_{1}-\hat{y}_{2}\right\|_{\infty} \\
\leq \frac{L \sqrt{2} \alpha^{1 / 4}}{\min _{\vartheta \in[-h, 0]} \lambda(\vartheta)}\left\|\hat{y}_{1}-\hat{y}_{2}\right\|_{\infty} .
\end{gathered}
$$

Consequently, the operator $A$ is the contracting operator on $\Omega$. Then by using the method of successive approximations [8] and by using the equality $\|A f-f\|_{\infty}=O\left(\alpha^{1 / 4}\right)$, we find

$$
\|y(\cdot, \alpha, D)-f(\cdot, \alpha, D)\|_{\infty}=O\left(\alpha^{1 / 4}\right) .
$$

Theorem 2. Let the assumptions of Theorem 1 be satisfied. Then the components of solution of the boundary value problem (2.7), (2.8) are defined by the asymptotic formulas

$$
\begin{gathered}
x_{s}(\vartheta, \varphi, \alpha)=\mathbb{S}_{s}(\vartheta, \alpha, \varphi) \Delta(\varphi)+\delta_{s 1} \frac{k}{r}\left(r-\frac{\varphi^{\prime}(\vartheta)}{\varphi(\vartheta)}\right)+O\left(\alpha^{1 / 4} ; \vartheta, \varphi\right), \\
\vartheta \in[-h, 0], \quad s=\overline{1,4},
\end{gathered}
$$

where

$$
\mathbb{S}_{s}(\vartheta, \alpha, \varphi)=(\sqrt{2} / 2) \sum_{j=1}^{2} e_{j}^{s-2} \exp \left(\alpha^{-1 / 4} e_{j} \int_{0}^{\vartheta} \lambda(\tau) d \tau\right) \lambda^{s-1}(\vartheta)
$$

and

$$
\Delta(\varphi)=\varphi(-h)-(k / r)\left(r-\varphi^{\prime}(0) / \varphi(0)\right) .
$$

P r o o f. By using the asymptotic representations of the components of solution (2.12) and the change of variables (2.10), we find the components of solution of system (2.7)

$$
\begin{gathered}
x_{s}(\vartheta, \alpha)=\sum_{j=1}^{4} e_{j}^{s-1} \lambda^{s-1}(\vartheta) \exp \left(\alpha^{-1 / 4} e_{j} \int_{\vartheta_{j}}^{\vartheta} \lambda(\tau) d \tau\right) D_{j}+\delta_{s 1} \frac{k}{r}\left(r-\frac{\varphi^{\prime}(\vartheta)}{\varphi(\vartheta)}\right) \\
+O\left(\alpha^{1 / 4} ; \vartheta, D_{1}, \ldots, D_{4}\right), \quad s=\overline{1,4}
\end{gathered}
$$

By substituting the obtained asymptotic representations into the boundary conditions (2.8), we have the system of algebraic equations

$$
\begin{aligned}
e_{3} D_{3}+e_{4} D_{4}+O\left(\alpha^{1 / 4} ; D_{1}, \ldots, D_{4}\right) & =0 \\
e_{3}^{2} D_{3}+e_{4}^{2} D_{4}+O\left(\alpha^{1 / 4} ; D_{1}, \ldots, D_{4}\right) & =0 \\
e_{1}^{3} D_{1}+e_{2}^{3} D_{2}+O\left(\alpha^{1 / 4} ; D_{1}, \ldots, D_{4}\right) & =0 \\
D_{1}+D_{2}+O\left(\alpha^{1 / 4} ; D_{1}, \ldots, D_{4}\right) & =\Delta(\varphi) .
\end{aligned}
$$

The last system for $\alpha=0$ has a unique solution. Then by taking into account the asymptotics of equations of the system (2.18), we find

$$
\begin{array}{ll}
D_{1}=\frac{\sqrt{2}}{2} e_{2} \Delta(\varphi)+O\left(\alpha^{1 / 4}\right), & D_{2}=\frac{\sqrt{2}}{2} e_{1} \Delta(\varphi)+O\left(\alpha^{1 / 4}\right), \\
D_{3}=O\left(\alpha^{1 / 4}\right), & D_{4}=O\left(\alpha^{1 / 4}\right) .
\end{array}
$$

By substituting the found values of constants into (2.17), we obtain (2.16). 


\section{Dependence of the Regularization Parameter on the Admissible Error}

Since $U x_{\alpha}=\chi_{\alpha}$, it follows that the discrepancy equation $(U x-\varphi, U x-\varphi)=\delta^{2}$ acquires the form

$$
\delta^{2}=\left(\chi_{\alpha}(0, \varphi)-\varphi(0)\right)^{2}+\int_{-h}^{0}\left(\chi_{\alpha}(s, \varphi)-\varphi(s)\right)^{2} d s .
$$

By using the variables $x_{2}$ and $x_{4}$ in (2.5), we have

$$
\left(\chi_{\alpha}(\vartheta, \varphi)-\varphi(\vartheta)\right)^{2}=\frac{4 k^{2} P^{2} x_{4}^{2}(\vartheta, \alpha, \varphi)}{r^{2} \varphi^{2}(\vartheta)} \alpha^{1 / 2}-\frac{4 k^{3} P^{3} x_{4}^{3}(\vartheta, \alpha, \varphi)}{r^{3} \varphi^{4}(\vartheta)} \alpha^{3 / 4}+O(\alpha ; \vartheta, \varphi) .
$$

By taking into account the formulas (2.16), we find

$$
\begin{aligned}
x_{4}(\vartheta, \alpha, \varphi) & =\frac{\sqrt{2}}{2} \lambda^{3}(\vartheta) \Delta(\varphi) \sum_{j=1}^{2} e_{j}^{2} \exp \left(\alpha^{-1 / 4} e_{j} \int_{0}^{\vartheta} \lambda(\tau) d \tau\right)+O\left(\alpha^{1 / 4} ; \vartheta, \varphi\right), \\
x_{4}^{2}(\vartheta, \alpha, \varphi) & =\frac{\lambda^{6}(\vartheta) \Delta^{2}(\varphi)}{2} \sum_{k, j=1}^{2} e_{k}^{2} e_{j}^{2} \exp \left(\alpha^{-1 / 4}\left(e_{k}+e_{j}\right) \int_{0}^{\vartheta} \lambda(\tau) d \tau\right) \\
& +\sqrt{2} \lambda^{3}(\vartheta) \Delta(\varphi) \sum_{j=1}^{2} e_{j}^{2} \exp \left(\alpha^{-1 / 4} e_{j} \int_{0}^{\vartheta} \lambda(\tau) d \tau\right) O\left(\alpha^{1 / 4} ; \vartheta, \varphi\right)+O\left(\alpha^{1 / 2} ; \vartheta, \varphi\right), \\
x_{4}^{3}(\vartheta, \alpha, \varphi) & =\frac{\sqrt{2}}{4} \lambda^{9}(\vartheta) \Delta^{3}(\varphi) \sum_{k, j, m=1}^{2} e_{k}^{2} e_{j}^{2} e_{m}^{2} \exp \left(\alpha^{-1 / 4}\left(e_{k}+e_{j}+e_{m}\right) \int_{0}^{\vartheta} \lambda(\tau) d \tau\right)+O\left(\alpha^{1 / 4} ; \vartheta, \varphi\right) .
\end{aligned}
$$

Hence $x_{4}(0)=O\left(\alpha^{1 / 4} ; \varphi\right)$ and $\left(\chi_{\alpha}(0, \varphi)-\varphi(0)\right)^{2}=O(\alpha ; \varphi)$. By taking into account the written above asymptotic formulas in $(3.1)$, we obtain

$$
\int_{-h}^{0}\left(\chi_{\alpha}(s, \varphi)-\varphi(s)\right)^{2} d s=\alpha^{3 / 4} \frac{2 k^{2} P^{2}}{r^{2}} \Delta^{2}(\varphi) \sum_{k, j=1}^{2} \frac{e_{j}^{2} e_{k}^{2}}{e_{j}+e_{k}} \frac{\lambda^{5}(0)}{\varphi^{2}(0)}+O(\alpha ; \varphi) .
$$

As a result the discrepancy equation acquires the form

$$
\delta^{2}=\alpha^{3 / 4} \gamma(\varphi)+O(\alpha ; \varphi)
$$

where $\gamma(\varphi)=\sqrt{2} \Delta^{2}(\varphi) \sqrt{r P^{3 / 2} \varphi(0) / k}$.

For $\Delta(\varphi) \neq 0$ the obtained equation has a unique continuous solution for a small positive $\delta$, which is determined by the formula

$$
\alpha(\delta, \varphi)=\gamma^{-4 / 3}(\varphi) \delta^{8 / 3}+O\left(\delta^{10 / 3}, \varphi\right) .
$$

Theorem 3. Let $\varphi \in W_{\infty}^{2}[-h, 0]$. Then the values of the regularizing operator for the equation $U x=\varphi$ on the set $D=\left\{\varphi: \Delta(\varphi) \neq 0, \varphi \in W_{\infty}^{2}[-h, 0]\right\}$ are given by the asymptotic formula

$$
R(\varphi, \delta)(\vartheta)=\mathbb{S}(\vartheta, \delta, \varphi) \Delta(\varphi)+\frac{k}{r}\left(r-\frac{\varphi^{\prime}(\vartheta)}{\varphi(\vartheta)}\right)+O\left(\delta^{2 / 3} ; \vartheta, \varphi\right), \quad \vartheta \in[-h, 0],
$$

where

$$
\mathbb{S}(\vartheta, \delta, \varphi)=(\sqrt{2} / 2) \sum_{j=1}^{2} e_{j}^{-1} \exp \left(\delta^{-2 / 3} \gamma^{1 / 3}(\varphi)\left(1+O\left(\delta^{2 / 3}, \varphi\right)\right) e_{j} \int_{0}^{\vartheta} \lambda(\tau) d \tau\right)
$$


P r o o f. A possible minimizing element corresponding to $\varphi \in W_{\infty}^{2}[-h, 0]$ is defined by the asymptotic formula

$$
x_{\alpha}(\vartheta, \varphi)=\mathbb{S}_{1}(\vartheta, \alpha, \varphi) \Delta(\varphi)+\frac{k}{r}\left(r-\frac{\varphi^{\prime}(\vartheta)}{\varphi(\vartheta)}\right)+O\left(\alpha^{1 / 4} ; \vartheta, \varphi\right), \quad \vartheta \in[-h, 0] .
$$

It can be shown, that the sufficient condition [8, p. 125] for the minimum of the functional $M^{\alpha}[\varphi, \cdot]$ is fulfilled. By substituting (3.2) into (3.4) we find the asymptotic formulas for the values of the regularized operator on the set $D$.

Let us introduce an operator $R_{1}: W_{\infty}^{2} \rightarrow H$ by the formulas

$$
R_{1}(\varphi)(\vartheta)=\frac{k}{r}\left(r-\frac{\varphi^{\prime}(\vartheta)}{\varphi(\vartheta)}\right), \quad \vartheta \in[-h, 0), \quad R_{1}(\varphi)(0)=\varphi(-h)
$$

and an operator $R_{2}: W_{\infty}^{2} \rightarrow H$ by the formulas

$$
\begin{aligned}
& R_{2}(\varphi, \delta)(\vartheta)=\frac{\sqrt{2}}{2} \sum_{j=1}^{2} e_{j}^{-1} \exp \left(\gamma^{1 / 3}(\varphi) \delta^{-2 / 3} e_{j} \int_{0}^{\vartheta} \lambda(\tau) d \tau\right) \Delta(\varphi)+R_{1}(\varphi)(\vartheta), \quad \vartheta[-h, 0), \\
& R_{2}(\varphi, \delta)(0)=R_{2}(\varphi, \delta)(-0) .
\end{aligned}
$$

Theorem 4. Let the assumptions of Theorem 2 be satisfied. Then $R_{1}: D \rightarrow H$ and $R_{2}: D \rightarrow$ $H$ are regularizing operators for the equation $U x=\varphi$.

P r o o f. The set of $\varphi_{p}$ for which the exact solutions $x_{p}$ of equation $U x=\varphi_{p}$ exist is everywhere dense in $H$ [9]. The inequalities

$$
\begin{gathered}
\left\|R_{1}\left(\varphi_{\delta}\right)-x_{p}\right\| \leq\left\|R_{1}\left(\varphi_{\delta}\right)-R\left(\varphi_{\delta}, \delta\right)\right\|+\left\|R\left(\varphi_{\delta}, \delta\right)-x_{p}\right\|, \\
\left\|R_{2}\left(\varphi_{\delta}, \delta\right)-x_{p}\right\| \leq\left\|R_{2}\left(\varphi_{\delta}, \delta\right)-R\left(\varphi_{\delta}, \delta\right)\right\|+\left\|R\left(\varphi_{\delta}, \delta\right)-x_{p}\right\| .
\end{gathered}
$$

hold for an arbitrary approximation $\varphi_{\delta}$ to $\varphi_{p}$ satisfying the conditions $\varphi_{\delta} \in D$ and $\left\|\varphi_{\delta}-\varphi_{p}\right\|_{2} \leq \delta$.

By using the formula (3.3), we obtain the differences

$$
R\left(\varphi_{\delta}, \delta\right)(0)-R_{1}\left(\varphi_{\delta}\right)(0)=O\left(\delta^{2 / 3} ; \varphi_{\delta}\right)
$$

and

$$
\begin{gathered}
R\left(\varphi_{\delta}, \delta\right)(\vartheta)-R_{1}\left(\varphi_{\delta}\right)(\vartheta)=\frac{\sqrt{2}}{2} \sum_{j=1}^{2} e_{j}^{-1} \exp \left(\delta^{-2 / 3}\left(\gamma^{1 / 3}\left(\varphi_{\delta}\right)+O\left(\delta^{2 / 3}\right)\right) e_{j} \int_{0}^{\vartheta} \lambda(\tau) d \tau\right) \Delta\left(\varphi_{\delta}\right) \\
+O\left(\delta^{2 / 3} ; \vartheta, \varphi_{\delta}\right), \quad \vartheta \in[-h, 0) .
\end{gathered}
$$

Let us estimate the integral

$$
\begin{gathered}
\int_{-h}^{0}\left(R_{1}\left(\varphi_{\delta}\right)(s)-R\left(\varphi_{\delta}, \delta\right)(s)\right)^{2} d s \\
=\frac{1}{2} \sum_{p, q=1}^{2} e_{p}^{-1} e_{q}^{-1} \Delta^{2}\left(\varphi_{\delta}\right) \int_{-h}^{0} \exp \left(\delta^{-2 / 3}\left(\gamma^{1 / 3}\left(\varphi_{\delta}\right)+O\left(\delta^{2 / 3}\right)\right) e_{j} \int_{0}^{s} \lambda(\tau) d \tau\right) d s .
\end{gathered}
$$

Since

$$
\left(e_{p}+e_{q}\right) \int_{0}^{s} \lambda(\tau) d \tau \neq 0
$$


for $p, q=1,2, s \in[-h, 0]$, then the equality is valid

$$
\int_{-h}^{0}\left(R_{1}\left(\varphi_{\delta}\right)(s)-R\left(\varphi_{\delta}, \delta\right)(s)\right)^{2} d s=O\left(\delta^{2 / 3} ; \varphi_{\delta}\right) .
$$

As a result, we have the asymptotic estimate

$$
\left\|R_{1}\left(\varphi_{\delta}\right)-R\left(\varphi_{\delta}, \delta\right)\right\|=O\left(\delta^{2 / 3}, \varphi_{\delta}\right) .
$$

In a similar way we find that

$$
\left\|R_{2}\left(\varphi_{\delta}, \delta\right)-R\left(\varphi_{\delta}, \delta\right)\right\|=O\left(\delta^{2 / 3}, \varphi_{\delta}\right) .
$$

By taking into account the resulting estimates, the property of the regularizing operator $R$, and inequality (3.5) we complete the proof of the theorem.

\section{Asymptotic of regularized solutions}

Let $H_{l o c}$ be the space of functions defined on the half-line $(-\infty,-h]$ restriction of which to any finite interval $\left[t^{-},-h\right], t^{-}<-h$, is the Hilbert space $H_{t^{-}}$with inner product

$$
\langle x, y\rangle_{t^{-}}=\int_{t^{-}}^{-h} y(s) x(s) \mu(d s)
$$

where $\mu(s)=s / h+m, s \in[(m-1) h, m h), m \leq-1, \mu(-h)=-1$. For an arbitrary solution $x_{m}(\vartheta, \varphi, \delta), \vartheta \in[-h, 0], m \leq-1$, of the finite-difference equation (1.2), consider the function $x(\cdot, \varphi, \delta) \in H_{l o c}$ defined by the formulas $x(m h+\vartheta, \varphi, \delta)=x_{m}(\vartheta, \varphi, \delta), \vartheta \in(-h, 0], m \leq-1$.

Let $x_{p}(\cdot)$ be en exact solution of system (1.1) on the interval $\left[t^{-},-h\right]$, corresponding to the initial function $\varphi_{p} \in D$. The relations

$$
\begin{aligned}
& \left\|x\left(\cdot, \varphi_{\delta}, \delta\right)-x_{p}(\cdot)\right\|_{t^{-}}=\left\langle x\left(\cdot, \varphi_{\delta}, \delta\right)-x_{p}(\cdot), x\left(\cdot, \varphi_{\delta}, \delta\right)-x_{p}(\cdot)\right\rangle_{t^{-}}^{1 / 2}=\left(\int_{t^{-}}^{-h}\left(x\left(s, \varphi_{\delta}, \delta\right)-x_{p}(s)\right)^{2} \mu(d s)\right)^{1 / 2} \\
& \leq \sum_{j=1}^{N}\left(\int_{-r}^{0}\left(x_{-j}\left(\vartheta, \varphi_{\delta}, \delta\right)-x_{p}(-j h+\vartheta)\right)^{2} \mu(d(-j h+\vartheta))\right)^{1 / 2} \\
& =\sum_{j=1}^{N}\left\|x_{-j}\left(\cdot, \varphi_{\delta}, \delta\right)-x_{p}(-j h+\cdot)\right\|=\sum_{j=1}^{N}\left\|R\left(x_{-j+1}\left(\cdot, \varphi_{\delta}, \delta\right), \delta\right)(\cdot)-x_{p}(-j h+\cdot)\right\| .
\end{aligned}
$$

hold for an arbitrary approximation $\varphi_{\delta} \in D$ to the initial function.

Here $N$ coincides with an integer part of the number $\left|t^{-}\right| / h,\|\varphi\|=(\varphi, \varphi)^{1 / 2}$. For the regularizing operator $R$ the last sum can be made arbitrarily small. Consequently, in the problem of finding solutions of the system (1.1) for any $t^{-}<-h$ the map $D \rightarrow H_{t^{-}}$defined by the formula $\varphi \rightarrow x(\cdot, \varphi, \delta)$ is regularizing. The functions $x(\cdot, \varphi, \delta) \in H_{l o c}$ are referred to as regularized solutions of the system (1.1) on the negative half-line.

For the initial function $\varphi \in W_{\infty}^{N+1}[-h, 0], N \geq 2$, we introduce the sequence of functions

$$
\varphi_{m}(\vartheta)=\frac{k}{r}\left(r-\frac{\varphi_{m+1}^{\prime}(\vartheta)}{\varphi_{m+1}(\vartheta)}\right), \quad m=\overline{-N,-1}, \quad \varphi_{0}(\vartheta)=\varphi(\vartheta), \quad \vartheta \in[-h, 0] .
$$

By using this sequence, we define new sequences

$$
x_{m}^{1}(\vartheta, \varphi)=R_{1}\left(\varphi_{m+1}\right)(\vartheta), \quad m=\overline{-N,-1}, \quad \vartheta \in[-h, 0],
$$




$$
x_{m}^{2}(\vartheta, \varphi, \delta)=R_{2}\left(\varphi_{m+1}, \delta\right)(\vartheta), \quad m=\overline{-N,-1}, \quad \vartheta \in[-h, 0] .
$$

Let us introduce the functions $x^{1}(\cdot, \varphi), x^{2}(\cdot, \varphi, \delta) \in H_{t^{-}}$, by the formulas

$$
\begin{gathered}
x^{1}(t, \varphi)=x_{m}^{1}(t-m h, \varphi), \quad x^{2}(t, \varphi, \delta)=x_{m}^{2}(t-m h, \varphi, \delta), \quad t \in((m-1) h, m h], \quad m=\overline{-N+1,-1}, \\
x^{1}(t, \varphi)=x_{m}^{1}(t-m h, \varphi), \quad x^{2}(t, \varphi, \delta)=x_{m}^{2}(t-m h, \varphi, \delta), \quad t \in\left[t^{-},-N h\right] .
\end{gathered}
$$

Theorem 5. Let $\varphi \in W_{\infty}^{N+1}[-h, 0]$. Then $\varphi \rightarrow x^{1}(\cdot, \varphi)$ and $\varphi \rightarrow x^{2}(\cdot, \varphi, \delta)$ from $\left\{\varphi: \Delta\left(\varphi_{m}\right) \neq 0\right\} \rightarrow H_{t^{-}}, m=-\frac{\infty}{-N+1,-1}$, are regularizing mappings for the Cauchy problem to the system (1.1) on the interval $\left[t^{-},-h\right]$.

P r o o f. Let $x_{p}(\cdot)$ be an exact solution of the system (1.1) on the interval $\left[t^{-},-h\right]$, corresponding to theinitial function $\varphi_{p}$. The inequalities

$$
\begin{gathered}
\left\|x^{1}\left(\cdot, \varphi_{\delta}\right)-x_{p}(\cdot)\right\|_{t^{-}}=\left(\int_{t^{-}}^{-h}\left(x^{1}\left(s, \varphi_{\delta}\right)-x_{p}(s)\right)^{2} \mu(d s)\right)^{1 / 2} \\
\leq \sum_{j=1}^{N}\left(\int_{-h}^{0}\left(x_{-j}^{1}\left(\vartheta, \varphi_{\delta}\right)-x_{p}(-j h+\vartheta)\right)^{2} \mu(d s)\right)^{1 / 2} \\
=\sum_{j=1}^{N}\left\|x_{-j}^{1}\left(\cdot, \varphi_{\delta}\right)-x_{p}(-j h+\cdot)\right\|=\sum_{j=1}^{N}\left\|R_{1}\left(\varphi_{-j+1}\right)(\cdot)-x_{p}(-j h+\cdot)\right\|, \\
\left\|x^{2}\left(\cdot, \varphi_{\delta}, \delta\right)-x_{p}(\cdot)\right\|_{t^{-}}=\left(\int_{t^{-}}^{-h}\left(x^{2}\left(s, \varphi_{\delta}, \delta\right)-x_{p}(s)\right)^{2} \mu(d s)\right)^{1 / 2} \\
\leq \sum_{j=1}^{N}\left(\int_{-h}^{0}\left(x_{-j}^{2}\left(\vartheta, \varphi_{\delta}, \delta\right)-x_{p}(-j h+\vartheta)\right)^{2} \mu(d(-j h+\vartheta))\right)^{1 / 2} \\
=\sum_{j=1}^{N}\left\|x_{-j}^{2}\left(\cdot, \varphi_{\delta}, \delta\right)-x_{p}(-j h+\cdot)\right\|=\sum_{j=1}^{N}\left\|R_{2}\left(\varphi_{-j+1}, \delta\right)(\cdot)-x_{p}(-j h+\cdot)\right\| .
\end{gathered}
$$

hold for an arbitrary approximation $\varphi_{\delta}$ to $\varphi_{p}$ satisfying the conditions $\varphi_{\delta} \in\left\{\varphi: \Delta\left(\varphi_{m}\right) \neq 0\right\}$, $m=\overline{-N+1,-1}$ and $\left\|\varphi_{\delta}-\varphi_{p}\right\| \leq \delta$.

For the regularizing operators $R_{1}$ and $R_{2}$ the last sum can be made arbitrary small. The proof of the theorem is complete.

The functions $x^{1}(\cdot, \varphi) \in H_{t^{-}}$and $x^{2}(\cdot, \varphi, \delta) \in H_{t^{-}}$are referred to as asymptotic regularized solutions of the system (1.1) on the interval $\left[t^{-},-h\right]$.

\section{Example}

In the report [2] one can find the following statistic data of the population size of elk in the Vologda region

$\begin{array}{cccccccccc}\text { year } & 1999 & 2000 & 2001 & 2002 & 2003 & 2004 & 2005 & 2006 & 2007 \\ \text { number of elks } & 22050 & 22320 & 23130 & 20000 & 20530 & 22850 & 26200 & 28750 & 32150\end{array}$

For the analysis of these data we use the obtained results of the paper. Firstly, we solve an identification problem for the Hutchinson-Wright equation. The breeding age of elks $h=2.5[10$, 
p. 231]. For the finding of the Malthusian factor $r$ and the capacity of the habitat $k$ we use the formula

$$
N(t)=N\left(t_{0}\right) \exp \left(r \int_{t_{0}}^{t}\left(1-\frac{\varphi(s-h)}{k}\right) d s\right), \quad t \in\left[t_{0}, t_{0}+h\right] .
$$

We choose the initial moment $t_{0}=2004$, and we approximate the initial function $\psi$ on the interval $[2001.5,2004]$ by cubic splines. Then, by using the above statistical data we obtain

$$
\psi(t)= \begin{cases}\frac{10}{39}\left(135690+76739 t+30789 t^{2}+3421 t^{3}\right), & t \in[2001.5,2002], \\ -\frac{10}{39}\left(-85674-1715 t+6723 t^{2}+2831 t^{3}\right), & t \in[2002,2003], \\ \frac{10}{39}\left(89115+12038 t+3600 t^{2}+610 t^{3}\right), & t \in[2003,2004] .\end{cases}
$$

We find the factors $r$ and $k$ from the equations

$$
\begin{aligned}
& N(2005)=N(2004) \exp \left(r \int_{2004}^{2005}\left(1-\frac{\psi(s-2.5)}{k}\right) d s\right), \\
& N(2006)=N(2004) \exp \left(r \int_{2004}^{2006}\left(1-\frac{\psi(s-2.5)}{k}\right) d s\right) .
\end{aligned}
$$

As a result of the numerical solution of this system of nonlinear algebraic equations we obtain $r=2.427$ and $k=21400$.

Let us show how we solve the problem of reconstruction of prehistory of the population size on the interval $[1999,2004]$. For a new value of the initial moment $t_{0}=2006.5$ and a new function $\varphi$ approximated by cubic splines on the interval [2004,2006.5] we have the identified model

$$
\dot{N}(t)=2.427\left(1-\frac{1}{21400} N(t-2.5)\right) N(t) .
$$

So, the initial function is defined by the formulas

$$
\varphi(t)= \begin{cases}-\frac{10}{39}\left(-89115-12038 t-3600 t^{2}+2573 t^{3}\right), & t \in[2004,2005], \\ \frac{10}{39}\left(83997+27392 t-11754 t^{2}+2545 t^{3}\right), & t \in[2005,2006], \\ -\frac{10}{39}\left(-113733+17212 t-10548 t^{2}+1172 t^{3}\right), & t \in[2006,2006.5] .\end{cases}
$$

By following the posed approach in this work, on the first step of applying the method of reconstruction of the prehistory we find $\gamma(\varphi)=970.173, \Delta(\varphi)=2459.48$. Then the value of the first regularizing operator is defined by the formulas

$$
R_{1}(\varphi)(t)= \begin{cases}\frac{21400.6(-2.29388+t)\left(18.4693+7.15864 t+t^{2}\right)}{(-1.82855+t)\left(21.575+7.9294 t+t^{2}\right)}, & t \in[2004,2005), \\ \frac{21400.6(3.72797+t)\left(11.8098-2.08253 t+t^{2}\right)}{(4.09592+t)\left(11.3947-1.21439 t+t^{2}\right)}, & t \in[2005,2006), \\ \frac{21400.6(-6.55253+t)\left(14.6794+3.81644 t+t^{2}\right)}{(-6.10399+t)\left(16.5387+4.60399 t+t^{2}\right)}, & t \in[2006,2006.5) .\end{cases}
$$


On the second step we obtain $\gamma(\varphi)=252.37, \Delta(\varphi)=-1254.41$, and

$$
R_{1}(\varphi)(t)=\left\{\begin{array}{l}
21400.6(-2.55816+t)(-1.56402+t)\left(16.7092+6.65895 t+t^{2}\right)(23.651 \\
\left.+8.42885 t+t^{2}\right) /\left((-2.29388+t)(-1.82855+t)\left(18.4693+7.15864 t+t^{2}\right)\right. \\
\left.\times\left(21.575+7.9294 t+t^{2}\right)\right), \quad t \in[2004,2005), \\
21400.6(3.48096+t)(4.34313+t)\left(12.3607-2.59903 t+t^{2}\right)(11.2027 \\
\left.-0.698089 t+t^{2}\right) /\left((3.72797+t)(4.09592+)\left(11.8098-2.08253 t+t^{2}\right)\right. \\
\left.\times\left(11.3947-1.21439 t+t^{2}\right)\right), \quad t \in[2005,2006), \\
21400.6(-6.81369+t)(-5.84275+t)\left(13.7488+3.31375 t+t^{2}\right)(17.793 \\
\left.+5.10659 t+t^{2}\right) /\left((-6.55253+t)(-6.10399+t)\left(14.6794+3.81644 t+t^{2}\right)\right. \\
\left.\times\left(16.5387+4.60399 t+t^{2}\right)\right), \quad t \in[2006,2006.5) .
\end{array}\right.
$$

The figure shows the results of computation for the value $\delta=10^{-2}$ of the admissible error. The graph of the modeled initial function is given by the black line and the graph of the asymptotic regularized solution $x^{2}(\cdot, \delta, \varphi)$ by the grey line, and the statistical data is given by black points.

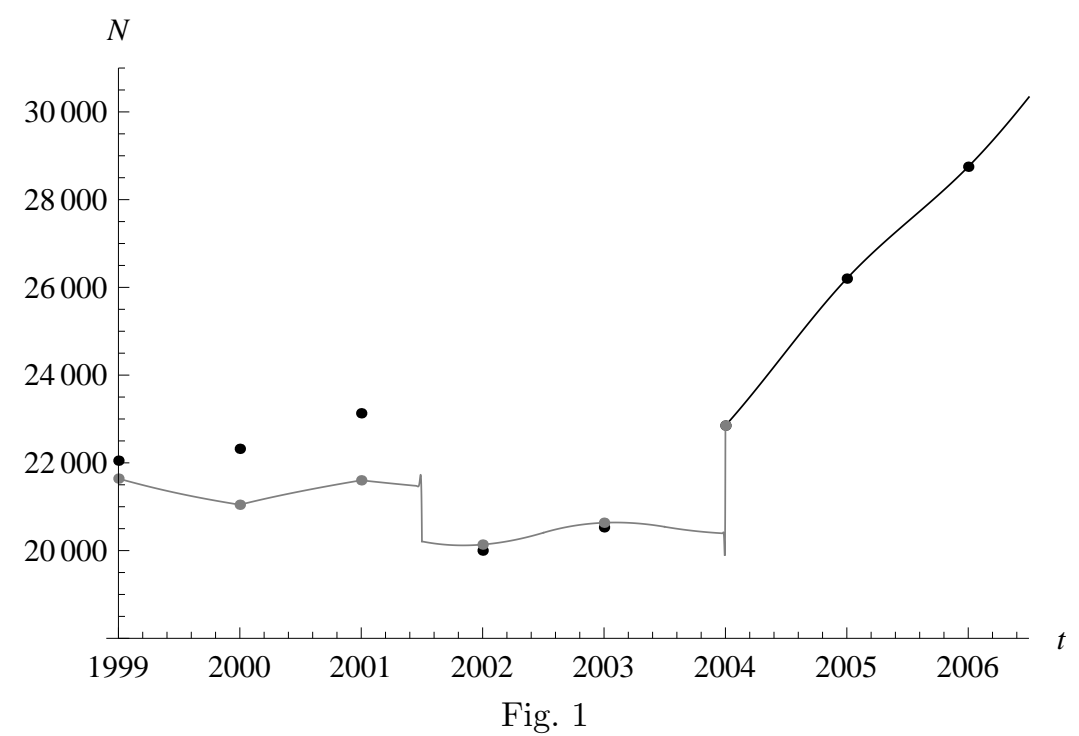

The error of reconstruction of the prehistory is defined by the formula

$$
\delta_{N}=\frac{1}{k} \sqrt{\sum_{j=1999}^{2003}\left(x^{2}(j, \delta, \varphi)-N(j)\right)^{2}}=0.11 .
$$

\section{References}

1. Lack D. The Natural Regulation of Animal Number. London: Oxford University Press. 1954. $343 \mathrm{p}$.

2. Report on the state and protection of the environment of the Vologda region in 2006 / Government of the Vologda region, the Department of environment and natural resources of the Vologda region. Vologda, 2007. 222 p. (in Russian) 
3. Mishchenko E.F., Kolesov Yu.S., Kolesov A.Yu. and Rozov N.Kh. Asymptotic methods in singularly perturbed systems, Monogr. Contemp. Math., Consultants Bureau, New York, 1994. $281 \mathrm{p}$.

4. Hassard B.D., Kazarinov N.D., and Wan Y.H. Theory and Application of the Hopf Bifurcation. Cambridge University Press, Cambridge, 1981. $320 \mathrm{p}$

5. Shampine L.F., Gladwell I., Thompson S. Solving ODEs with MATLAB PDF. Cambridge University Press, 2003. 272 p.

6. Tikhonov A.N., Arsenin V.Y. Solutions of Ill-Posed Problems, Winston, New York, 1977. $258 \mathrm{p}$.

7. Vainberg M.M. Variational Method and Method of Monotone Operators. John Wiley and Sons, New York, 1974. 356 p.

8. Kantorovich L.V. and Akilov G.P. Functional Analysis. Pergamon Press, New York, NY, 1982. $604 \mathrm{p}$.

9. Hale J.K. and Verduyn-Lunel S.M. Introduction to Functional Differential Equations. Applied Mathematical Sciences. Springer-Verlag New York, 1993. Vol. 99. 450 p.

10. Danilkin A.A. Reindeer (Cervidae). Moscow.: GEOS, 1999. 552 p. (in Russian) 